\title{
Single-mode whispering gallery lasing from metal-clad GaN nanopillars
}

\author{
K. H. Li, Zetao Ma, and H. W. Choi* \\ Department of Electrical and Electronic Engineering, The University of Hong Kong, China \\ *Corresponding author: hwchoi@hku.hk \\ Received October 7, 2011; revised November 23, 2011; accepted December 9, 2011; \\ posted December 12, 2011 (Doc. ID 156115); published January 24, 2012
}

\begin{abstract}
An ordered hexagonal closed-packed nanopillar array is fabricated on GaN. A metal coating is then applied to encapsulate the pillars for promoting optical confinement within the cylindrical cavity. Room-temperature lasing at $373 \mathrm{~nm}$ is observed under pulsed excitation, at a lasing threshold of $0.42 \mathrm{MW} / \mathrm{cm}^{2}$. With pillar diameters of around $980 \mathrm{~nm}$, the number of modes overlapping the emission spectrum is reduced, giving rise to single-mode whispering gallery stimulated emission. Finite-difference time-domain simulations are carried out for the prediction of resonant frequencies and electric field patterns corresponding to the resonant modes. () 2012 Optical Society of America OCIS codes: $140.3945,140.4780,140.5960,230.5750,220.4241$.
\end{abstract}

Over the past decade, blue and ultraviolet (UV) nitridebased edge-emitting laser diodes (LDs) have been adopted for various critical industrial and medical applications, such as optical data storage, high-resolution printing, biomedical instrumentation, and fluorescence sensing. To meet the growing demand for nitride LDs, more attention has been paid to developing a verticalcavity surface-emitting laser (VCSEL) [1,ㄹ]. The nitride VCSEL structure requires a pair of high reflectivity distributed Bragg reflectors (DBRs) on either side of the epitaxial structure to form a vertical Fabry-Perot (F-P) cavity in order for resonant modes to be established. While the top dielectric DBR can readily be deposited, the bottom DBR has to be epitaxially grown concurrently. Typically a large number of pairs of nitride-based alloys is inserted beneath the laser structure to achieve the desired reflectivity. In [3], a crack-free cavity sandwiched between lattice-matched AlInN/GaN DBRs was demonstrated, with a reflectivity higher than 99\%. Another approach incorporates two dielectric DBRs [ $\underline{4}]$ on both faces of a nitride thin film, enabled by removing the sapphire substrate through laser lift-off (LLO) [5]. This approach is not without shortcomings; the highenergy laser pulses may cause damage to the active region, and the thickness of the cavity cannot be precisely controlled due to the roughened surface. The establishment of a circular cavity that supports whispering gallery (WG) modes is yet another approach; such cavities can generally be fabricated by a single pattern-and-etch process $[6,7]$. The resonant frequency and mode spacing are simply determined by the dimension of the disk-shape element. Typically, microdisk structures of micrometer dimensions are defined by photolithography, resulting in multiple mode lasing [8]. Nanosphere lithography (NSL) as adopted in this work is a highly efficient and low-cost nanopatterning solution capable of fabricating ordered hexagonal nanopillar arrays. Pillar structures of submicron dimensions have larger mode spacing, which is suitable for minimizing the number of modes overlapping the gain spectrum, thus achieving single-mode stimulated emission.

We report on the fabrication and optical properties of metal-clad GaN nanopillar arrays in this Letter. A twodimensional silica nanosphere monolayer was assembled by spin-coating. The coated layer acts as a hard lithographic mask for pattern transfer to the GaN layer, resulting in etched pillar structures. The additional metal cladding layer serves to enhance optical confinement effects, promoting the establishment of WG modes.

NSL is employed to fabricate a large-area ordered hexagonal nanopillar array, and the process flow is described in detail in our earlier work [9]. Silica spheres with a mean diameter of $990 \mathrm{~nm}$ are spin-coated onto a wafer, whose structure consists of InGaN/GaN multiquantum wells (MQWs) sandwiched between $n$-GaN and $p$-GaN layers, grown by metal-organic chemical vapor deposition on $c$-plane sapphire. The periodical nanosphere pattern is transferred to GaN by inductively coupled plasma etching. After sphere mask removal, a metal coating is deposited over the nanopillar array using electron beam evaporation. The optical properties of the nanopillars are evaluated by conducting room-temperature (r.t.) photoluminescence (PL) measurements. A Spectra-Physics diode-pumped solid state (DPSS) $349 \mathrm{~nm}$ laser is used as an excitation source, with 4 ns pulse duration and 1 $\mathrm{kHz}$ repetition rate. Instead of exciting PL from the $p$ $\mathrm{GaN}$ side, the focused beam with spot diameter of $60 \mu \mathrm{m}$ was incident perpendicularly through the bottom polished sapphire surface since the metal layer strongly attenuates the incident UV beam. The PL signal was collected with a fiber probe at a detection angle of $\sim 80^{\circ}$ to the normal, dispersed by an Action SP2500A $500 \mathrm{~mm}$ spectrograph, and coupled to a PI-PIXIS open-electrode CCD; the system offered optical resolutions of $0.04 \mathrm{~nm}$.

Figure 1(a) shows a field-emission scanning electron microscopy (FE-SEM) image of a hexagonal close-packed nanosphere monolayer array assembled using spincoating. The coated sample was dry etched for $180 \mathrm{~s}$, giving an etch depth and a pillar diameter of approximately $750 \mathrm{~nm}$ and $980 \mathrm{~nm}$, respectively, evident from Fig. 1(b) and (c). Point defects and line dislocations are inevitable using self-assembly methods. To ensure that the air-gaps between neighboring pillars are fully filled with metal, a higher deposition rate of around $3 \AA$ /s was used. The pillars were almost completely covered by the metal clad, as shown in Fig. 1(d).

Figure 2(a) shows the r.t. PL spectra of four nanopillar cavity samples without and with different metal 

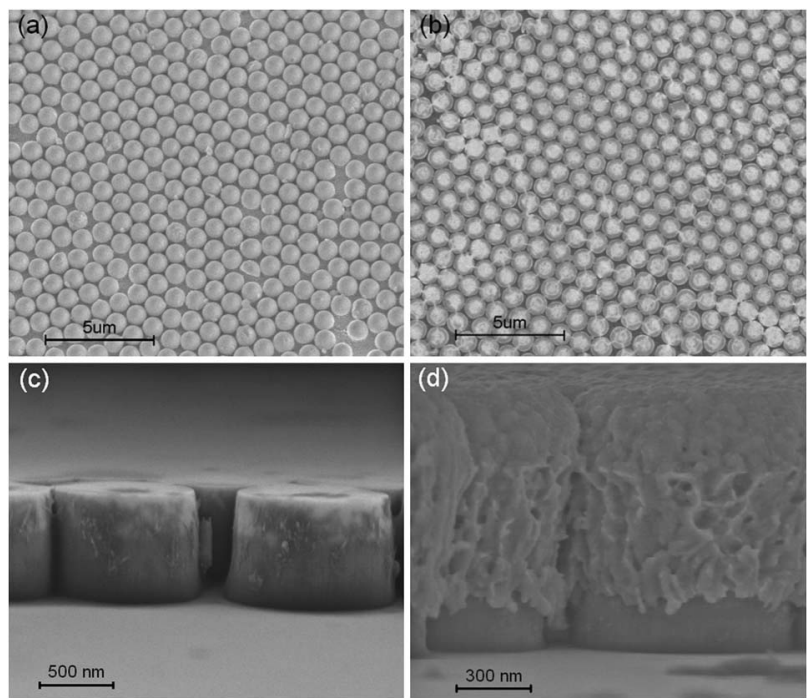

Fig. 1. FE-SEM images showing (a) ordered hexagonal monolayer arrays of spheres; (b) pillar array with etch depth and pillar diameter of $750 \mathrm{~nm}$ and $980 \mathrm{~nm}$, respectively; and cross-sectional views of pillars (c) before and (d) after metal coating.

claddings — namely $\mathrm{Au}, \mathrm{Ag}$, and $\mathrm{Al}$ - under an excitation density of $\sim 0.85 \mathrm{MW} / \mathrm{cm}^{2}$. Two broadband emissions centered at $\sim 366.43 \mathrm{~nm}$ and $\sim 433.07 \mathrm{~nm}$ were observed from the nanopillar sample, corresponding to spontaneous emissions from GaN and InGaN/GaN MQWs. The intensity of MQW emission is much lower than that of GaN emission because the excitation beam was incident through the substrate side. A significant portion of the incident photons has been absorbed by the GaN layer before propagating to the MQWs region, resulting in domination of GaN UV emission. To promote resonant modes within the nanopillar, $\mathrm{Au}, \mathrm{Ag}$, and $\mathrm{Al}$ metal layers are coated onto the surface to encapsulate the cavities. From the PL spectra, a resonant mode was observed only from the Al-coated sample, while the other samples experienced mild PL enhancements. The Al coating, with the highest UV reflectivity, is thus demonstrated to be capable of enhancing the confinement effects of a pillar cavity. Figure 2(b) shows excitation-varying PL spectra from the Al-coated sample. Two spectral peaks centered at

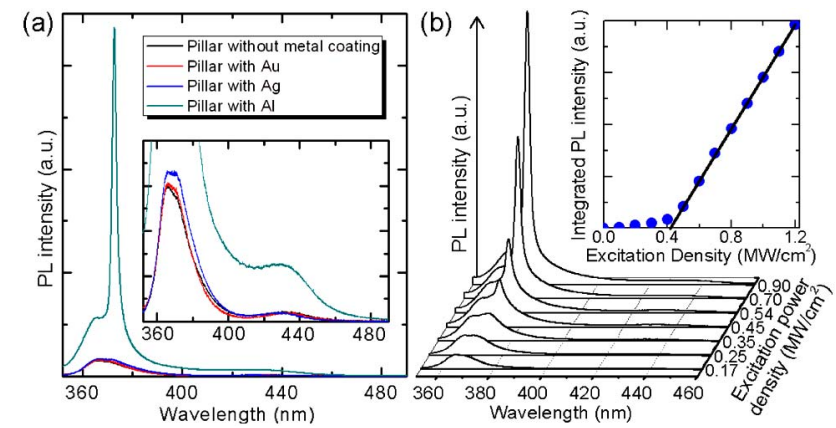

Fig. 2. (Color online) (a) R.t. PL spectra of nanopillars with various metal coatings at high excitation power density of $\sim 0.85 \mathrm{MW} / \mathrm{cm}^{2}$. (b) PL spectra of Al-coated nanopillars under varying excitation power density, with lasing mode observed at $373 \mathrm{~nm}$; the inset shows integrated PL intensity versus pump power density. $\sim 365 \mathrm{~nm}$ and $\sim 373 \mathrm{~nm}$ can be resolved. However, when the excitation density exceeds threshold (extracted from the plot to be $\sim 0.42 \mathrm{MW} / \mathrm{cm}^{2}$ ), the latter peak becomes dominant. The plot in the inset of Fig. 2(b) verifies the nonlinear increase in PL intensity above threshold, a characteristic of stimulated emission. Figure 3(a) and (b) show captured CCD images of the PL spot below and above threshold, respectively. Above threshold, specklelike patterns are clearly observed, attributed to coherent emission.

While the geometries of nanopillars support WG modes, the presence of a metal coating may also introduce vertical F-P modes. To distinguish between the mechanisms, an $\mathrm{Al}$ layer was coated onto an as-grown wafer for comparison. Both the nanopillar and as-grown samples are then subjected to UV laser excitation at $0.85 \mathrm{MW} / \mathrm{cm}^{2}$, the PL spectra of which are plotted in Fig. 3(c), indicating that no optical modes can be observed from the Al-coated as-grown sample; the likelihood of F-P mode lasing is therefore slim. With a single mirror coating, photons can still escape from the opposite GaN/sapphire or sapphire/air interfaces, resulting in inefficient optical confinement. On the other hand, pillar cavities with metal coating on both the sidewall and the top surface provide strong lateral confinement within the circular cavity. This is particularly so for $\mathrm{Al}$, which provides a high reflectivity in the UV spectral region, according to the reflectivity curves for the corresponding metals [10] shown in the inset of Fig. 3(c). For reference, with a lower UV reflectivity, the Ag-clad nanopillars would also lase at a higher threshold of $0.97 \mathrm{MW} / \mathrm{cm}^{2}$.

Finite-difference time-domain (FDTD) simulations are performed to verify the resonant characteristics of the nanopillars with different metal claddings. The computational area and the mesh size $(\Delta x)$ are fixed at $1.5 \times$ $1.5 \mu \mathrm{m}^{2}$ and $5 \mathrm{~nm}$, respectively, with the time step set to 0.00967 fs to satisfy the Courant stability condition of $\Delta t_{\max }=(\Delta x / c \sqrt{2})=0.0118$ fs. The simulated transverse electric (TE) mode spectra for the $\mathrm{Al}, \mathrm{Ag}$, and $\mathrm{Au}$ cladded nanopillars in the wavelength range of 400 to $500 \mathrm{~nm}$ are plotted in Fig. 4(a). In the spectrum of the Al-clad pillars, a first-order mode at $\sim 365 \mathrm{~nm}$ and a second-order mode at $\sim 373 \mathrm{~nm}$ are observed, overlapping with the peak of the GaN emission spectrum. Three consecutive first-order modes observed at 347,365 , and
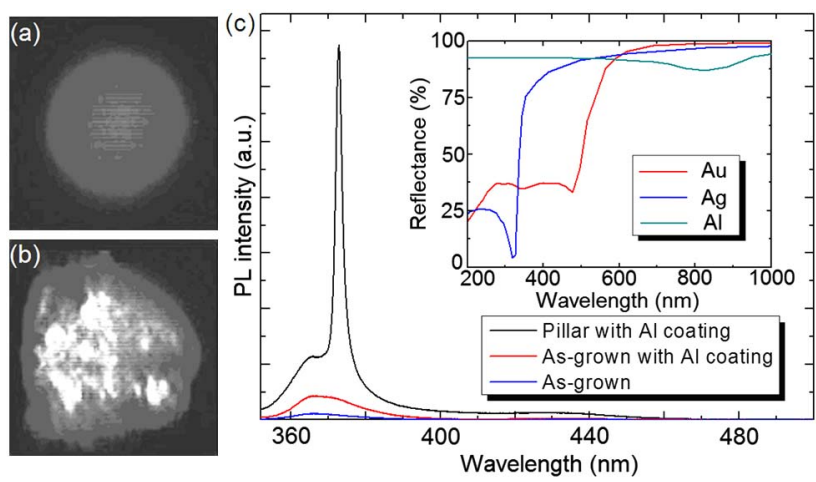

Fig. 3. (Color online) CCD-captured images of PL emission from Al-clad pillar (a) below and (b) above threshold. (c) Effects of $\mathrm{Al}$ coating on as-grown and nanopillar samples; the inset shows the reflectance curves for $\mathrm{Au}, \mathrm{Ag}$, and $\mathrm{Al}$. 


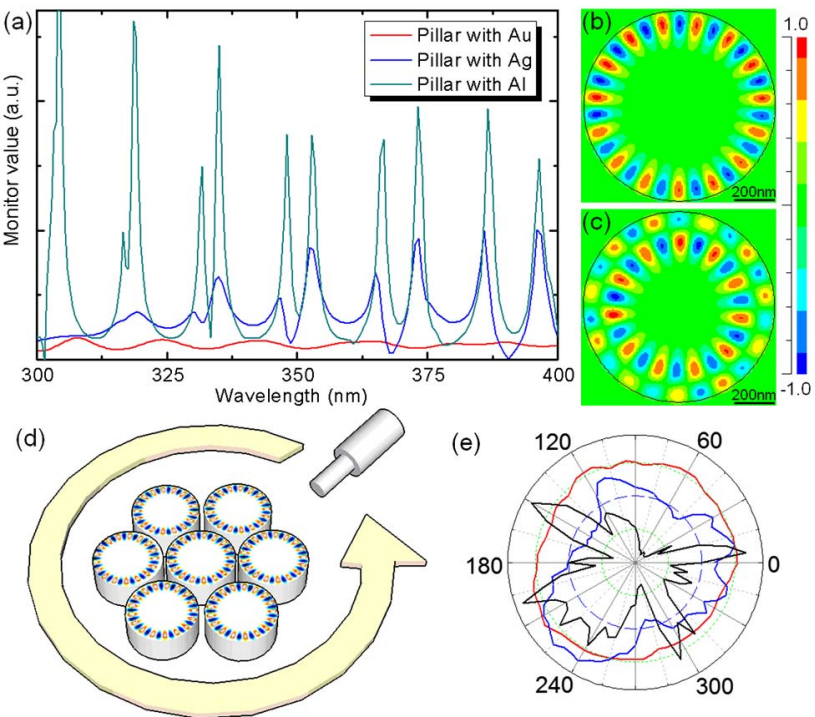

Fig. 4. (Color online) (a) FDTD-simulated spectra showing resonant characteristics of metal-clad nanopillar cavities; FDTDcomputed steady-state electric field patterns of the resonant modes at (b) $365 \mathrm{~nm}$ and (c) $373 \mathrm{~nm}$. (d) Schematic diagram showing the setup for measuring in-plane angular emission pattern. (e) Angular emission patterns of Al-clad pillars from the in-plane (black) and vertical (blue) directions. The curve in red corresponds to in-plane measurement from an as-grown sample.

$386 \mathrm{~nm}$ were consistent with the relation $\Delta \lambda_{\mathrm{WG}}=$ $\lambda^{2} / 2 \pi R n$ [11], where $R$ is the pillar radius and $n$ is the effective refractive index (of 2.3). Also note that its modal intensities, thus optical confinement, remain high across the frequency range of interest, while those for Ag-clad pillars decline with reducing wavelengths, whereas no resonant modes are observed for the Au-clad pillars. This is consistent with the reflectivities of the respective metal coatings as plotted in the inset of Fig. 3(c). The FDTD-simulated steady-state electric field patterns corresponding to the resonant modes at $365 \mathrm{~nm}$ and $373 \mathrm{~nm}$ are shown in Fig. 4(b) and (c), respectively, neglecting coupling effects. It can be seen that the field corresponding to the first-order mode at $365 \mathrm{~nm}$ is concentrated around the periphery of the pillar, and therefore would be prone to scattering losses along the roughened plasma-etched sidewalls [12]. On the other hand, the field pattern for the higher-order mode at $373 \mathrm{~nm}$ is established within the pillar and will not suffer from optical losses, explaining why this mode becomes the dominant lasing mode above threshold.

With WG emission modes, a highly directional emission pattern can be expected in the planar directions [13]. To verify this, the angular-resolved PL in the near-planar direction is measured from a pillar array by rotating a fiber around the central axis of the sample, with the collection angle maintained at $\sim 80^{\circ}$ to the normal, as illustrated in Fig. 4(d). A high degree of emission directionality is clearly present, with multiple intensity peaks at various discrete angles, deduced from the curve in black from the polar plots of Fig. 4(e). As the emission modes are scattered out of the cavities through roughened (etched) sidewalls, the recorded pattern is asymmetrical; geometrical imperfections of individual pillars and nonuniformities between pillars adds to the asymmetry. Such periodicity is clearly absent from either the angularresolved PL pattern, as measured in the near-normal direction (blue curve), or the as-grown sample (red curve).

A final note on the cold-cavity quality factor $Q$, which has been evaluated as 150 at an excitation power density of $0.4 \mathrm{MW} / \mathrm{cm}^{2}$. The relatively low magnitude may be attributed to sidewall scattering losses, intracavity coupling, and, most importantly, the absence of a gain region. However, the low gain is merely a limitation of the PL measurement geometry. In the electroluminescent device under development, emission from the MQWs will be dominant, providing sufficient gain to overcome the losses. Nevertheless, the present optical-pumped demonstration verifies the feasibility of this approach for forming optical microcavities.

In summary, we have demonstrated the fabrication of metal-clad nanopillar structures using NSL. Pillars with $\mathrm{Al}$ coating provide strong lateral confinement, and r.t. lasing at $373 \mathrm{~nm}$ has been observed, at a lasing threshold of $0.42 \mathrm{MW} / \mathrm{cm}^{2}$. The lasing mechanism is identified as a WG mode. Single-mode lasing is possible due to the submicron dimensions of individual pillars.

This work was supported by a GRF grant by the Research Grant Council of Hong Kong (project HKU $7118 / 09 \mathrm{E})$.

\section{References}

1. Y. Higuchi, K. Omae, H. Matsumura, and T. Mukai, Appl. Phys. Express 1, 121102 (2008).

2. T. C. Lu, C. C. Kao, H. C. Kuo, G. S. Huang, and S. C. Wang, Appl. Phys. Lett. 92, 141102 (2008).

3. J. F. Carlin, J. Dorsaz, E. Feltin, R. Butté, N. Grandjean, and M. Ilegems, Appl. Phys. Lett. 86, 031107 (2005).

4. J. Y. Zhang, L. E. Cai, B. P. Zhang, X. L. Hu, F. Jiang, J. Z. Yu, and Q. M. Wang, J. Lightwave Technol. 27, 55 (2009).

5. W. S. Wong, T. Sands, N. W. Cheung, M. Kneissl, D. P. Bour, P. Mei, L. T. Romano, and N. M. Johnson, Appl. Phys. Lett. 75, 1360 (1999).

6. S. Chang, N. B. Rex, R. K. Chang, G. Chong, and L. J. Guido, Appl. Phys. Lett. 75, 166 (1999).

7. D. Simeonov, E. Feltin, H. J. Bühlmann, T. Zhu, A. Castiglia, M. Mosca, J.-F. Carlin, R. Butté, and N. Grandjean, Appl. Phys. Lett. 90, 061106 (2007).

8. H. W. Choi, K. N. Hui, P. T. Lai, P. Chen, X. H. Zhang, S. Tripathy, J. H. Teng, and S. J. Chua, Appl. Phys. Lett. 89, 211101 (2006).

9. K. H. Li and H. W. Choi, J. Appl. Phys. 110, 053104 (2011).

10. D. R. Lide, CRC Handbook of Chemistry and Physics, 82nd ed. (CRC Press, 2001).

11. G. F. Neumark, I. Kuskovsky, and H. X. Jiang, Wide Bandgap Light-Emitting Materials and Devices (Wiley, 2007).

12. A. C. Tamboli, E. D. Haberer, R. Sharma, K. H. Lee, S. Nakamura, and E. L. Hu, Nat. Photon. 1, 61 (2007).

13. V. M. Apalkov and M. E. Raikh, Phys. Rev. B 70, 195317 (2004). 